Fernández Leyva, H. y Pérez Marqués, C. M. ${ }^{a}$ (2018). La situación de Cuba en las investigaciones obre disponibilidad léxica, en RILEX. Revista sobre investigaciones léxicas, 1/II, pp. 31-51.

\title{
LA SITUACIÓN DE CUBA EN LAS INVESTIGACIONES SOBRE DISPONIBILIDAD LÉXICA
}

\section{THE SITUATION OF CUBA IN THE INVESTIGATIONS ON LEXICAL AVAILABILITY}

\author{
Haydeé Fernández Leyva \\ Universidad de Oriente \\ haydee@uo.edu.cu
}

\author{
Celia María Pérez Marqués \\ Universidad de Oriente \\ celiapm@uo.edu.cu
}

\section{RESUMEN}

Dentro de los estudios lexicales a comunidades lingǘsticas, los de disponibilidad léxica han cobrado auge en las últimas décadas, especialmente en la española a partir del llamado de López Morales con la coordinación del Proyecto panhispánico de disponibilidad léxica (PPHDL). Valiosas y numerosas han sido las respuestas en toda la extensión de la Península Ibérica e Hispanoamérica. Sin embargo, fundamentalmente por dificultades materiales, los avances de Cuba son incipientes. Dada la dispersión en áreas geográficas, momentos de realización, publicaciones en eventos y el hecho de que algunos se encuentran inéditos, el objetivo de este trabajo es ofrecer una panorámica de los estudios de disponibilidad léxica (DL) realizados en Cuba (2011-2017) para aquilatar los avances del país en este tipo de investigación. Para cumplirlo se relacionan 26 investigaciones, que constituyen la totalidad de las que se tiene conocimiento. Entre ellas, se encuentran trabajos de diplomas, tesis de maestría y artículos científicos que demuestran la labor que se está realizando en distintas provincias del territorio cubano.

Palabras clave Cuba, disponibilidad léxica, vocabulario disponible, español, lexicoestadística

\begin{abstract}
Within the lexical studies of linguistic communities, those of lexical availability have gained momentum in the last decades, especially in the Spanish one since the call of López Morales with the coordination of the Pan-Hispanic Project of Lexical Availability (PPHDL). Valuable and numerous have been the answers in all the extension of the Iberian Peninsula and Hispano-America. However, fundamentally due to material difficulties, Cuba's advances are incipient. Given the dispersion in geographic areas, moments of studies, publications in events and the fact that some are unpublished, the objective of this work is to provide an overview of the studies of lexical availability (DL) carried out in Cuba (2011-2017) to assess the progress of this country in this type of research. To fulfill it, 26 investigations are related, which constitute the totality about which we have knowledge. Among them, there are works of diplomas, master's thesis and scientific articles that demonstrate the work that is being done in different provinces of the Cuban territory.
\end{abstract}

Keywords: Cuba, lexical availability, available vocabulary, Spanish, lexical statistics 


\section{INT'RODUCCIÓN}

A partir de la convocatoria realizada por López Morales para la concepción del vocabulario disponible del mundo hispánico, los países de esta lengua han dirigido parte de los esfuerzos de las investigaciones lingüísticas para lograr este propósito. Si bien algunos de ellos llevan una tradición en esta línea, Cuba todavía se encuentra en una etapa de germinación, aunque con ánimo creciente por cambiar esa realidad.

Dada la dispersión en áreas geográficas, momentos de realización, publicaciones en eventos y el hecho de que algunos se encuentran inéditos, el objetivo de este trabajo es ofrecer una panorámica de los estudios de disponibilidad léxica (DL) realizados en Cuba (2011-2017) para aquilatar los avances del país en este tipo de investigación.

En este territorio, las acciones a favor de la disponibilidad léxica pudiéramos datarlas del 2000, fecha a partir de la cual se produjeron varios eventos que propiciarían su desarrollo.

En un análisis sobre el estado de estas investigaciones en lengua española, Bartol (2006, p. 383) menciona la inserción de la isla en esta línea con la coordinación de Gregori, directora del Instituto de Literatura y Lingüística José Antonio Portuondo Valdor y miembro de la Academia Cubana de la Lengua. Sin embargo, por dificultades materiales, la intención no se consolidó.

Almeida (2012, pp. 6-7) refiere que en 2007 ve la luz el proyecto La enseñanza del español en el mundo hispánico, coordinado por López Morales y representado en el país por González Mafud, profesora de la Universidad de La Habana y miembro de la Academia Cubana de la Lengua. Este proyecto estuvo dirigido a reconocer y mejorar el estado de los métodos de enseñanza-aprendizaje del español como lengua materna y tenía, como líneas de estudio, la riqueza y disponibilidad léxicas, madurez sintáctica, cohesión y coherencia discursivas.

Entre las actividades del mismo, estuvo la organización en 2011, por parte de la Academia Cubana de la Lengua, del seminario La enseñanza de la lengua materna. El tema correspondiente a la DL fue impartido por Bartol, quien expuso los orígenes, concepto, metodología y aplicaciones de estos estudios. 
Todos estos elementos condicionaron la toma de conciencia de la necesidad de estas investigaciones y que se buscaran alternativas para desarrollarlas.

\section{LAS INVESTIGACIONES CUBANAS}

En el país existen fundamentalmente tres instituciones que desde su papel de formadores actúan como nodo de irradiación para los estudios lingüísticos: la Universidad de La Habana, la Universidad Central Marta Abreu de Las Villas y la Universidad de Oriente. La ubicación geográfica de estos centros, occidente, centro y oriente, de modo respectivo, ha propiciado que se desarrollen los estudios de DL en las provincias lindantes a estas universidades, para así paulatinamente ir desarrollando este tema de investigación a lo largo de todo el territorio cubano.

Por otro lado, los servicios que se prestan en el país para la enseñanza del español como lengua extranjera han condicionado que los lingüistas se interesen por comprobar la incidencia del proceso de enseñanza en el vocabulario disponible de sus alumnos. De este modo, comparten con aquellos que evalúan el vocabulario disponible de informantes de lengua materna la aplicación pedagógica de estas investigaciones.

\subsection{ESTUdIOS CUBANOS DE DISPONIBILIDAD LÉXICA EN ESPAÑOL COMO LENGUA MATERNA}

La acción que concretó el desarrollo de la DL en Cuba se obtuvo a partir de la estancia realizada por Pérez Marqués, en 2010, como profesora visitante en la Universidad de Málaga (Ávila \& Villena, 2010, 10), sitio en el que se nutrió de la metodología de esta escuela y le permitió procesar los datos de encuestas que había aplicado en el 2000 a escolares del municipio santiaguero Guamá. Como resultado, publicó el primer artículo cubano sobre el tema, titulado "Disponibilidad léxica de los escolares de Guamá en tres centros de interés" (2011). Entre los méritos del estudio está el haber tenido en cuenta el impacto de la Batalla de Ideas ${ }^{1}$ sobre el pensamiento creador de los estudiantes, cuestión que se refleja en el índice de disponibilidad (ID) alcanzado por algunos

\footnotetext{
1 Acción politica de la Revolución Cubana donde se desarrolla un debate de carácter ético en defensa de sus avances en la justicia social, de la integridad nacional y el internacionalismo. Se acompaña por un conjunto de programas (más de doscientos) que se llevan a cabo en las diferentes esferas de la sociedad y que han contribuido a la realización de importantes cambios positivos en la calidad de vida de los cubanos (Ecured, 2018).
} 
vocablos referentes a la introducción de las nuevas tecnologías en el sector. En él la autora da a conocer los resultados de un estudio de DL a escolares entre tercero y sexto grado de ese municipio santiaguero, en los centros de interés (1) la escuela, (2) la casa y (3) Los animales, y precisa la influencia de cinco variables externas en la DL de los informantes: sector (urbano/ rural), tipo de escuela (graduada/ multigrado), grado (tercero/ cuarto/ quinto/ sexto), sexo (masculino/ femenino) y nivel sociocultural y ocupacional de los padres (alto/ medio/ bajo). El análisis se centra en el índice de disponibilidad obtenido por los veinte vocablos más disponibles en cada centro de interés (CI).

De él derivaron las ponencias "Una visión regional a través de la disponibilidad léxica de los escolares del municipio Guamá" (2012), presentada en el evento Nuevos Talleres Internacionales de Estudios Regionales y Locales (Urbanos) III "La Región, sus Diversas Visiones" en 2012 en Santa Clara y "La disponibilidad léxica de los escolares del municipio Guamá como reflejo de su cultura local" (2014), socializada en la IX Conferencia Internacional sobre Lenguas Extranjeras, Comunicación y Cultura, WEFL A en 2014 en la Universidad de Holguín.

En 2012, Almeida defiende el trabajo de diploma titulado La enseñanza del español en el mundo hispánico. Disponibilidad léxica en escolares de La Habana, con el objetivo general de describir y analizar el léxico disponible de los estudiantes de 11 y 16 años de cuatro escuelas de la capital cubana. Las variables sociales empleadas fueron nivel educacional $\left(6^{\text {to }}\right.$ grado $/ 11^{\text {no }}$ grado) y prestigio institucional (escuela buena/escuela mala). Según expresa la autora $(2012,64)$, los factores para la selección de las escuelas, según esta última variable, fueron establecidos por el Ministerio de Educación (MINED). Y por su diversidad, no se explicitaron en el trabajo. No obstante, sean cuales fueren esos factores, se recomienda modificar la nominación de las variantes a un modo menos peyorativo, como por ejemplo, "escuela de alto prestigio institucional" y "escuela de bajo prestigio institucional". Los centros de interés estudiados corresponden con los determinados por el proyecto La enseñanza del español en el mundo hispánico son: partes del cuerpo bumano, animales, alimentos, medios de transporte, profesiones y oficios y accidentes geográficos. En ese trabajo, una vez obtenido el comportamiento interno del léxico de los 
estudiantes cubanos encuestados, se realiza una comparación de índole cuantitativa con los de Puerto Rico y República Dominicana, para conocer el estado de Cuba en relación con otros países caribeños miembros del proyecto. También se ofrecen algunas consideraciones sobre las relaciones de hiperonimia y sinonimia, identificadas en el léxico disponible, y un análisis de la influencia que el vocabulario de los libros de textos utilizados en el proceso de enseñanza-aprendizaje pudiera estar teniendo sobre el vocabulario disponible de los estudiantes. Entre las conclusiones, destaca la observación de que, desde el punto de vista cualitativo, existe una notable coincidencia en los estudiantes de primaria entre su vocabulario disponible y el de los libros de textos, lo que demuestra la influencia de los materiales didácticos en el repertorio léxico de estos niños, aun cuando hay vocablos que la autora considera que se dejan de presentar. Esto constituye un llamado a los educadores ante la necesidad de mantener actualizados los materiales didácticos en la medida en que varíe el vocabulario disponible de esas edades. Por otro lado, se expresa que no se corrobora la hipótesis de que las escuelas con mayor prestigio institucional han de tener los mejores resultados cuantitativos. Esto patentiza la necesidad de explicitar qué se entiende por escuelas "buenas" y "malas", para conocer cuáles son los criterios que están incidiendo sobre ese resultado. Entre los valores de la investigación destacan la obtención y el análisis cuantitativo y cualitativo del repertorio léxico del que disponen esos educandos. Además, su concepción metodológica permitió mantener una secuencia coherente entre el análisis léxico-estadístico y el proceso de enseñanza- aprendizaje del español en las escuelas analizadas.

Un año más tarde, como parte de un ejercicio de igual tipo, en la Universidad de Oriente, Sierra defiende la investigación Disponibilidad léxica de estudiantes preuniversitarios santiagueros (2013). Como declara su autora (2013, p. 3), el estudio sigue, en líneas generales, las pautas metodológicas del Proyecto Panhispánico de Disponibilidad Léxica y tiene como objetivo caracterizar cuantitativa y cualitativamente el léxico disponible de los estudiantes preuniversitarios del duodécimo grado del municipio Santiago de Cuba. Las variables sociales determinadas fueron sexo (masculino/ femenino), ubicación geográfica del centro de estudios (ciudad de Santiago de Cuba/ poblados de Santiago 
de Cuba), tipo de centro (general/ especializado), nivel de lectura (alto/ medio/ bajo) y nivel sociocultural de los padres (alto/ medio-alto/ medio-bajo/ bajo). Si bien la selección de las variables empleadas está en correspondencia con la de otros estudios realizados en España, al evaluar el nivel de lectura y el nivel sociocultural de los padres, la autora creó nuevos parámetros teniendo en cuenta la estructura de la sociedad cubana. Para la aplicación del test de disponibilidad se consideraron los 16 centros de interés tradicionales, con el fin de viabilizar futuras comparaciones. Además, se agregaron las artes y los deportes debido a que, entre las escuelas preuniversitarias seleccionadas, se encuentran una de enseñanza artística y otra deportiva. Debe destacarse que, además de realizar la caracterización cuantitativa y cualitativa del léxico disponible de los informantes, la autora valora las relaciones asociativas del léxico en los distintos centros de interés y la ortografía de las palabras recogidas en el test asociativo. Es una investigación que aporta los elementos fundamentales de un estudio de esta modalidad, de gran valor para los maestros de escuelas preuniversitarias santiagueras y, como trabajo de diploma, un referente digno de la disponibilidad léxica en Cuba.

En ese período, Perdomo et al. (2015) informan sobre los resultados del estudio de los errores ortográficos cometidos en el centro de interés la cocina y sus utensilios por 440 estudiantes de duodécimo grado durante el curso escolar 2013-2014 en la provincia Matanzas. El análisis permitió conocer que existe tendencia a cometer errores ortográficos en esta área temática, ya que 397 informantes presentaron alguna dificultad. Estas fueron: confusiones de sonidos alveolares representados por las grafías $l$ y $r$ : cardero(s) por caldero(s); de sonidos fricativos y africados representados por las grafías $c, z, s$ y $x$ : expumadera por espumadera; de sonidos palatales representados por las grafías $l l, \tilde{n}$, ch y $y$ : cuchicho por cuchillo; simplificación del sonido alveolar vibrante múltiple: arocera por arrocera; confusiones ortográficas entre $b / v$ : basos por vasos; entre $g / j$ : vagilla por vajilla; y entre $y / l l:$ rallador por rayador, supresión o ultracorrección de $h$ : ormilla por hornilla; y por último, la adición o supresión de sonidos: rocera por arrocera (Perdomo et al., 2015). Dados estos resultados, se analizaron los libros de textos y los programas de estudio de la asignatura Lengua materna en el nivel primario, lo que 
permitió concluir que no existen lecturas, vocabularios ni objetivos ortográficos definidos que incluyan a este contenido, lo que demuestra la necesidad de tener en cuenta este tema en la planificación de la enseñanza.

Igualmente, en 2015 Pérez et al. publican el artículo "Proyecto «Vocabulario disponible de los estudiantes de noveno grado en Santiago de Cuba»: un monitoreo de la efectividad del sistema educativo cubano desde la léxico-estadística" (2015) con el fin de divulgar el proyecto, que tiene como objetivo general conocer el vocabulario que los estudiantes de noveno grado de Santiago de Cuba emplearían ante determinadas temáticas, y como objetivos específicos:

1. Obtener el vocabulario disponible de estudiantes santiagueros de noveno grado.

2. Precisar los principales rasgos del vocabulario disponible de estos estudiantes.

3. Determinar la influencia de las variables extralingüísticas en la disponibilidad léxica de estos hablantes.

4. Precisar la disponibilidad de sus faltas ortográficas. (Pérez et al., 2015, pp. 63-64).

La muestra estuvo conformada por 56 mujeres y 53 hombres estudiantes de noveno grado, con edades acordes con el año que cursaban, nacidos y criados en la provincia de Santiago de Cuba y con padres o tutores que cumplieran esta misma condición o al menos hubieran vivido en la provincia desde hacía diez años. Se declararon cinco variables externas: ubicación geográfica del centro de estudios (ciudad Santiago de Cuba/ poblado Santiago de Cuba), sexo (masculino/ femenino), procedencia del informante (municipio Santiago de Cuba/ otro municipio de la provincia Santiago de Cuba), nivel sociocultural y ocupacional de los padres o tutores (alto/ medio/ bajo) y tipo de centro (de enseñanza general/ de arte/ deportivo).

Para la recogida de los datos, además de los 16 centros de interés tradicionales, se tuvieron en cuenta los mismos añadidos por Sierra (2013): artes y deportes, ya que para los investigadores resultaba de interés conocer la cultura de los estudiantes en correspondencia con el tipo de escuela.

El estudio está inscrito en el programa Problemas actuales del sistema educativo cubano. Perspectivas de desarrollo y se inserta como una tarea más en el proyecto sombrilla Monitoreo del Sistema Nacional de Educación, lo que demuestra la voluntad del Ministerio de Educación por perfeccionar el desarrollo léxico de los estudiantes. 
Para dar cumplimiento al cuarto objetivo específico del proyecto, dentro del marco de la IX Conferencia Internacional Lingüistica (2015), organizada por el Instituto de Literatura y Lingüística José Antonio Portuondo Valdor en La Habana, se presenta la comunicación: "Disponibilidad de errores ortográficos en estudiantes de noveno grado de Santiago de Cuba" (Pérez Marqués et al., 2015). Los autores partieron de la idea de que, al aplicar la metodología para la obtención de la DL y procesar con el programa Lexidisp los vocablos semi-lematizados, se debería obtener un listado de vocablos organizado en orden descendente de su índice de disponibilidad, en el cual, si un mismo vocablo ha sido producido con distintas formas ortográficas, debería aparecer en distintas posiciones de la lista en dependencia de la rapidez con que cada una de aquellas haya llegado a la mente de los informantes. De ahí que se pueda hablar de la existencia de una disponibilidad ortográfica, que incluye formas correctas e incorrectas (Pérez Marqués et al., 2015, p. 3). Se obtuvo que los centros de interés con mayores errores ortográficos fueron: (1) iluminación y medios de airar un recinto, (2) deportes y (3) objetos colocados sobre la mesa para la comida. También, de modo general, las palabras escritas correctamente alcanzaron una disponibilidad mayor, las faltas de ortografía más frecuentes fueron la acentuación, los cambios de letras, especialmente entre $s-c-q-$ $x$ y $b-v, y$ las omisiones de letras, además de que todas las variables externas consideradas influyeron en la disponibilidad, especialmente sexo, procedencia del informante y tipo de centro de estudios.

Los resultados generales del proyecto y el diccionario de frecuencias, El léxico Disponible de los Estudiantes de Noveno Grado en Santiago de Cuba, aunque todavía inéditos, se pueden localizar en el Centro de Lingüística Aplicada de esa ciudad.

En el evento mencionado anteriormente se presentaron además otras dos ponencias: "Léxico disponible de los escolares habaneros y matanceros en dos centros de interés: descripción, análisis y comparación” (2015) de Suardíaz y “La disponibilidad léxica de estudiantes de preuniversitario de Pinar del Rio, Cuba: Incidencia de la variable sexo" (2015) de Cabrera Albert et al.

Entre los objetivos de la primera se encuentra analizar el léxico disponible de escolares habaneros a partir de la organización y estandarización de las listas de 
disponibilidad léxica de 400 informantes en los centros de interés (1) alimentos y bebidas y (2) los animales y, en contraste con los resultados de los estudiantes matanceros para estos campos, describir el comportamiento de los extranjerismos en los listados de léxico disponible de los escolares habaneros y matanceros, a partir de los centros de interés analizados (Suardíaz, 2015, p. 3). Las variables sociales que se tomaron en cuenta fueron sexo (masculino/ femenino), nivel sociocultural (bajo/ medio/ alto) y ubicación del centro de estudios (centro/ periferia). Se concluyó que en La Habana los escolares aportaron 676 palabras más que los estudiantes matanceros, que en ambas provincias las mujeres tuvieron un mayor número de actualizaciones de unidades léxicas y que en la capital hay una mayor presencia de extranjerismos. Finalmente, se informa que en la Facultad de Artes y Letras están las encuestas editadas y codificadas, así como las tablas calculadas de ambas provincias a través de Dispolex, de modo que sirvan para futuras investigaciones.

En cuanto a la segunda ponencia presentada, su objetivo de trabajo fue como se señala:

valorar la incidencia de la variable sexo en el léxico disponible de una muestra de jóvenes pinareños para descubrir si las diferencias sociales entre hombres y mujeres, los papeles que se le asignan o desempeñan en la sociedad, conducen a actitudes diferentes en los encuestados (Cabrera Albert et al., 2015, p. 2).

La muestra estuvo conformada por 100 estudiantes, de ellos 43 mujeres y 57 hombres, estudiantes del último año de preuniversitario con edades comprendidas entre los $17 \mathrm{y}$ 18 años. La metodología empleada para la selección de los informantes y el procesamiento de los datos se ajustó a las directrices del Proyecto Panbispánico de Léxico Disponible, al que se inserta el trabajo. Como resultados se obtienen que los estudiantes de sexo masculino producen más vocablos en los cuatro centros de interés analizados: (1) partes del cuerpo humano, (2) alimentos y bebidas, (3) medios de transporte y (4) profesiones y oficios. Sin embargo, según los autores este aspecto podría considerarse como no concluyente por la señalada desproporción en el número de individuos. Por su significación para el comportamiento social, cabe destacar el análisis realizado en lo que concierne al tipo de vocabulario expresado por ambos sexos, como son la observación de un mayor empleo de disfemismos por parte de los hombres en el centro 
de interés (1) partes del cuerpo bumano, a la alta disponibilidad de bebidas alcohólicas en el vocabulario de (2) alimentos y bebidas, fundamentalmente en los hombres; así como la existencia de voces referentes a innovaciones criollas para palear la problemática del transporte en el país en los últimos treinta años. También, la marcada preferencia de profesiones en masculino por los hombres, y en femenino por las mujeres.

Este trabajo fue la base de un artículo publicado en 2017 bajo el título "Incidencia de la variable "sexo" en la disponibilidad léxica de estudiantes de preuniversitario en Pinar del Río, Cuba" (Cabrera Albert et al., 2017). Si bien para su desarrollo se utilizaron los datos anteriores, en esta publicación se observa un análisis cuantitativo más profundo, pues se tuvieron en cuenta los valores de frecuencia absoluta de cada centro de interés, el rango de productividad de respuestas y la aplicación de la prueba estadística $t$ de Student, que corroboró la no existencia de diferencias significativas entre la variabilidad y los promedios de palabras aportados por los informantes.

En 2016, Cárdenas defiende el trabajo de diploma titulado La disponibilidad léxica de los estudiantes de onceno grado del IPVCE Ernesto Guevara en la provincia de Villa Clara. Este estudio, al igual que el de Pérez et al (2015), también está inscrito en el programa nacional Problemas actuales del sistema educativo cubano. Perspectivas de desarrollo. Tiene como objetivo general: "describir el comportamiento de la disponibilidad léxica de los estudiantes de onceno grado del preuniversitario en función de los centros de interés" (Cárdenas, 2016, p. 5). La muestra la conformaron 30 alumnos, 15 hombres y 15 mujeres, que cursaban el onceno grado en el IPVCE Ernesto Guevara. Se seleccionó esa institución docente porque a ella acuden estudiantes de todos los municipios villaclareños. Las variables sociales consideradas fueron sexo y nivel sociocultural. Aunque el análisis de estas se realizó en los 16 centros de interés tradicionales, la autora solo presenta el correspondiente a (1) partes del cuerpo bumano, (2) la ropa, (3) partes de la casa, (4) los muebles de la casa, (5) alimentos y bebidas y (6) la escuela, por ser los que mayor cantidad de vocablos aportaron. También analiza el tipo de relaciones asociativas entre los vocablos informados. En cuanto a los centros de interés, se concluyó que (5) alimentos y bebidas y (6) la escuela son los que mayor cantidad de vocablos presentan, mientras que (4) los muebles de la casa es el más cohesionado. Con respecto de las 
variables sociales, se observó que existe un comportamiento similar entre la media de vocablos de los niveles socioculturales medio y alto, y que las mujeres aportan una media de palabras superior a los hombres. Por último, que las relaciones asociativas más recurrentes son las de hiperonimia, hiponimia y las motivadas por oposición. Con este ejercicio de culminación de estudios, la Universidad Central Marta Abreu de Las Villas se incorpora a las investigaciones de este tipo. Aunque todavía incipiente, pues no se presenta un diccionario de los vocablos con el índice de disponibilidad léxica organizado, se muestra la intención de desarrollar esta línea investigativa desde esa región del país.

En ese mismo año, se publica el artículo "Competencia comunicativa, disponibilidad léxica y lectura. Sus relaciones en la formación inicial de profesores de Español-Literatura" (2016), por Calzadilla y Domínguez, desarrollado a partir de la determinación de insuficiencias vinculadas con la disponibilidad léxica que repercuten en la competencia comunicativa de los profesores en formación inicial de esa materia y la comprobación de que entre sus causas figuran limitaciones con la lectura como estrategia de acceso al léxico. En él se presentan los fundamentos teóricos de la tesis de aspirantura a Doctor en Ciencias Pedagógicas, titulada La disponibilidad léxica de profesores de Español- Literatura en formación inicial, actualmente en elaboración por Calzadilla, profesora de la Universidad de Las Tunas.

En junio de 2017, como ejercicio de culminación de estudios, Alentado presenta: Análisis y descripción de la disponibilidad léxica de los escolares de seis institutos preuniversitarios habaneros en tres centros de interés (2017). La autora se traza entre los objetivos analizar el léxico disponible de los estudiantes habaneros de seis institutos preuniversitarios en los centros de interés (1) partes del cuerpo, (2) la ciudad y (3) el campo. El trabajo es una contribución al completamiento del análisis de la muestra tomada por Suardíaz (2015), pues solo se contemplan 121 estudiantes, de ellos 67 hombres y 54 mujeres. Las variables sociales atendidas fueron sexo (hombre/ mujer), carácter del centro de estudio (centro/ periferia) y nivel sociocultural (alto/ medio/ bajo). Además del análisis cuantitativo y cualitativo de los CI, la autora analiza las relaciones de sinonimia que se pueden establecer en las listas generales, los cubanismos existentes y compara 
los resultados con los de los estudiantes valencianos. Desde lo cuantitativo se concluyó que el centro de interés (1) partes del cuerpo agrupa la mayor cantidad de palabras y es el más cohesionado. También, que, al contrario de lo esperado en las hipótesis, los estudiantes de escuelas pertenecientes a municipios periféricos presentaron una mayor riqueza léxica en las áreas temáticas (2) la ciudad y (3) el campo, y que los hombres tuvieron mayor productividad de palabras y vocablos totales respecto a las mujeres. No obstante, este resultado, así como que los estudiantes de nivel sociocultural medio actualizarán la mayor cantidad de vocablos, todavía no es concluyente, puesto que la disparidad en la muestra influye de modo directo en los datos. Se observó que los estudiantes de nivel sociocultural alto presentaron los mayores valores de disponibilidad en (2) la ciudad y (3) el campo, mientras que los de nivel bajo alcanzaron resultados inferiores. Desde el punto de vista cualitativo, se observó que el sexo masculino produjo una mayor cantidad de voces tabú, aunque con bajos ID y grupos nominales relacionados con el género. Por otro lado, las mujeres anotaron un léxico exclusivo en (3) el campo, a partir de actualizaciones de nombres de flores. Se corroboró el empleo de la sinonimia; se documentaron 86 cubanismos, lo que representa un 12,18\% del total de vocablos y se comprobó cómo los CI (2) la ciudad y (3) el campo agrupan un vocabulario más diverso entre los estudiantes habaneros y matanceros.

En noviembre, en la X Conferencia Internacional Lingüistica (2017), Perdomo et al. presentan la comunicación "Disponibilidad léxica en el centro de interés "Partes del cuerpo" en la provincia de Matanzas", que tuvo como objetivos determinar el léxico disponible de estudiantes de duodécimo grado en el centro de interés y provincias mencionados, así como analizar la resemantización que han sufrido algunas palabras llamadas mal sonantes vinculadas con este tema. Los datos se obtuvieron de las encuestas aplicadas por la autora en 2013 (vid. supra). Entre los resultados cuantitativos generales se informa que el total de palabras obtenido es 7429 y un promedio de respuestas por informante de 16.9. Sobre la disponibilidad de las palabras mal sonantes se observó que la mayoría aluden a los órganos sexuales y que los varones superan a las hembras en cantidad de vocablos, aun en los referidos a los órganos femeninos. Como aportes del trabajo y de gran utilidad para estudios comparativos, además del análisis de las 
palabras mal sonantes informadas en el CI, se encuentra el vocabulario obtenido, procesado en Dispolex y ordenado por posición, índice de disponibilidad y frecuencias relativa y acumulada, además del porcentaje de aparición.

En el mismo evento, Oramas y García presentan el reporte parcial de un estudio comparado de disponibilidad léxica entre Cuba y México titulado "Estudio de disponibilidad léxica de estudiantes de tercer año de bachillerato en las ciudades de Matanzas y Morelia (DLM2)" (2017). En el artículo se informa sobre las etapas que constituyen la investigación: documental, teórica, instrumental y ejecutiva; y se menciona parte del análisis del vocabulario recogido en los CI (1) partes del cuerpo bumano, (2) el campo y (3) Internet. El análisis comparado refleja que entre el vocabulario de ambas ciudades hay un $82 \%$ de coincidencia de términos en (1) partes del cuerpo bumano, $58 \%$ en (2) el campo y $48 \%$ en Internet; y que existe la tendencia al uso de grupos nominales como una sola unidad léxica. También se muestran brevemente algunos vocablos que evidencian la variación diatópica en los CI (1) partes del cuerpo bumano y (2) el campo, así como el aprendido en el constructo histórico-cultural fuera de la escuela. Por último, los autores recomiendan líneas de trabajo didáctico orientadas al mejoramiento del proceso de enseñanza-aprendizaje en las clases de lengua y literatura.

\subsection{EsTUdios CUBANOS DE DISPONIBILIDAD LÉXICA EN ESPAÑOL COMO LENGUA EXTRANJERA}

A partir del año 2013 surgen trabajos en los que se relacionan la disponibilidad léxica y la enseñanza del español como lengua extranjera. Dicha producción se debe, fundamentalmente, al programa de maestría Enseñana del Español como Lengua Extranjera de la Universidad de Oriente y al desarrollo de esta línea investigativa en la Facultad de Español para No Hispanohablantes (FENHI) de la Universidad de La Habana.

Como resultado de la primera escuela, en diciembre de 2013 Fernández defiende la tesis de maestría La selección y ocurrencias del vocabulario por centros de interés en el manual Español para Todos 1. La autora, sobre la base de los datos informados en el Diccionario de Frecuencias del Léxico Disponible de Preuniversitarios Santiagueros (Sierra, 2013), se plantea como objetivo analizar el vocabulario presente en el manual didáctico Español para 
Todos 1, relacionado con temáticas específicas, así como sus ocurrencias en el texto, a partir de criterios léxico-estadísticos aplicables a la enseñanza de lenguas. Para cumplirlo, una vez determinados los presupuestos teóricos y metodológicos sobre la selección y ocurrencias del vocabulario en manuales para la enseñanza del español como lengua extranjera, procede a hacer un vaciado del manual, a la contabilización de las ocurrencias de los vocablos utilizados por centros de interés, a la comparación de su léxico con el disponible de los estudiantes preuniversitarios santiagueros, y al análisis de la presentación del vocabulario relativo a los centros de interés de DL a partir de la comprobación de si en el libro Español para Todos 1 están presentes vocablos disponibles y sus ocurrencias. Para determinar la cantidad de unidades léxicas que debe incluir cada área temática, Fernández asume como criterio regulador el promedio de vocablos por sujeto. Este es corregido en el artículo "Posible aplicación del índice de disponibilidad léxica a la selección del vocabulario de manuales de ELE” (2015), en el que, tras comparar el promedio de vocablos de los preuniversitarios santiagueros (Sierra, 2013) con el de veinte estudiantes canadienses que estudiaban español en la Universidad de Oriente; en la búsqueda de un corte inferior de vocablos al sugerido en la tesis de maestría, se basa en el cálculo recomendado por Gómez Devís (2004) y en la noción de prototipicidad de los vocablos empleada por Ávila \& Villena (2010) para sugerir la limitación de la cantidad de vocablos por centros de interés a partir del número de vocablos con un índice de disponibilidad $>0.1$ mencionados por al menos el $25 \%$ de los informantes, para garantizar que se empleen vocablos que formen parte de la categorización conceptual colectiva del centro de interés y corregir la sobrecarga léxica existente en el manual didáctico. La importancia de la investigación radica en que ha dado a conocer al colectivo de autores del manual Español para Todos 1 si el léxico empleado es el más útil y sus ocurrencias son las más indicadas para propiciar su mejor adquisición por parte de los estudiantes.

Por otro lado, entre los resultados de la FENHI deben mencionarse los artículos publicados por Cuba \& Cabrera Domescq (2013); Cabrera Domecq et al. (2015 a., 2015 b., 2017) y la tesis de maestría de Jia Chen (2016). 
El primero de ellos, titulado "El Léxico de los estudiantes no hispanohablantes de español. Estudios preliminares" (Cuba \& Cabrera Domescq 2013), contempla entre sus objetivos determinar el léxico disponible de los estudiantes de $2^{\text {do }}$ y $3^{\text {ro }}$ de la Licenciatura en Lengua Española para No Hispanohablantes de la FENHI en la Universidad de La Habana. En este artículo se analiza el léxico de los estudiantes sinohablantes a partir de los instrumentos estadísticos: pruebas de riqueza léxica y pruebas de disponibilidad léxica. Se trabajó con las hipótesis de que es mayor el léxico disponible en los estudiantes sinohablantes de $3^{\text {ro }}$ que el de los estudiantes de $2^{\text {do }}$ año, y que el léxico disponible de las estudiantes es mayor que el de los informantes masculinos, por lo que el sexo influye en la disponibilidad léxica de los estudiantes de la muestra. El test de disponibilidad abarcó los CI (1) la escuela, (2) el cuerpo bumano, (3) las comidas y bebidas, (4) la cindad y (5) profesiones y oficios. Fue aplicado a 19 mujeres y 9 varones de $2^{\text {do }}$ año, así como a 30 mujeres y 13 varones de $3^{\text {ro }}$. El análisis arrojó que, en cifras absolutas, los estudiantes de $3^{\text {ro }}$ dominan más palabras que los de segundo en tres áreas temáticas. Quedó refutada la primera hipótesis, al demostrarse que los estudiantes de $2^{\text {do }}$ año tienen mejores resultados que los de $3^{\text {ro }}$. También se corroboró que el promedio de vocablos de las hembras es mayor que el de los varones. Teniendo en cuenta que las diferencias de cantidades en la muestra influyen en este resultado, se sugiere revisarlo con cantidades iguales, o solo valorar aquellos parámetros en los que este factor no determine diferencias, como se hizo con los promedios de palabras y vocablos por centros de interés según el año, en los que $2^{\text {do }}$ superó a $3^{\text {ro }}$ en ambos casos.

En 2014 Xiau Huo y Siru Yun presentan su trabajo de diploma, cuyos resultados se socializaron, junto a la autoría de Cabrera Domecq, a través de los artículos "La disponibilidad léxica en estudiantes sinohablantes de ELE en la carrera de Medicina” (2015) e "Índice de disponibilidad léxica en estudiantes de español como segunda lengua" (2015). En ellos se establece como objetivo determinar los índices de disponibilidad léxica de estudiantes sinohablantes de la carrera de Medicina en la Facultad de Ciencias Médicas Julio Trigo. La investigación trabajó con estudiantes de $3^{\text {ro }}$ y $5^{\text {to }}$ años de la carrera, con un total de 38 informantes divididos en iguales 
cantidades según el género: 19 mujeres y 19 hombres. Del total de centros de interés tradicionales para los estudios de DL fueron escogidos: (1) el cuerpo bumano, (2) las comidas y bebidas, (3) la escuela, (4) la ciudad y (5) profesiones y oficios, porque, a juicio de las autoras, son los que más relación tienen con la vida de los estudiantes. De este modo se obtiene una panorámica general del comportamiento por áreas temáticas del número de vocablos y palabras aportados, sus promedios, porcentaje de aparición y un análisis por sexo.

También en ese año Fernández y Pérez en, "La disponibilidad léxica como recurso para el acercamiento a la identidad cultural en el aula de ELE” (2015), defienden la idea de que una de las vías para que un estudiante de español como lengua extranjera aprenda sobre la identidad cultural cubana es darle a conocer las palabras más útiles del contexto en que se encuentra inmerso; de este modo, el profesor debe buscar herramientas que le permitan seleccionar el contenido léxico que va a enseñar en el aula, el cual debe reflejar los rasgos generales que unen a Cuba al resto de la comunidad hispana, además de sus particularidades. Las autoras demuestran mediante ejemplos de los vocablos más disponibles en los centros de interés la ropa y medios de transporte ${ }^{2}$, obtenidos de informantes de Madrid y Santiago de Cuba, que la selección del vocabulario que debe ser enseñado en un aula de ELE basada en la variante de esta última ciudad, además de propiciar un conocimiento del mundo desde sus particularidades denotativas, no frena la comunicación.

En el curso escolar 2015-2016 Jia Chen defiende la tesis de maestría Análisis comparativo de la disponibilidad léxica en español como lengua extranjera entre estudiantes chinos y sudafricanos, y bajo el mismo título publica en 2017 los resultados principales. El trabajo tuvo como objetivo comparar la disponibilidad léxica de estudiantes de ELE de esas nacionalidades en la carrera de Medicina en La Habana. La muestra estuvo conformada por 38 estudiantes chinos e igual número de sudafricanos, que cursaban el $3^{\text {er }}$ año de Medicina. La única variable social con la que se operó fue sexo y los centros de interés analizados fueron los mencionados en Cabrera Domecq (2015a y 2015b), pues los datos de los estudiantes chinos procedían de ese estudio.

\footnotetext{
${ }^{2}$ Considerados entre los que mayores diferencias muestran en cuanto al vocabulario que contienen.
} 
En el trabajo se hizo necesario limitar el análisis de los datos matemáticos al total de unidades léxicas, rango de palabras y vocablos, promedio por informante, frecuencia de aparición de palabras por centro de interés, dado que los cálculos hubo que realizarlos manualmente (no se pudo utilizar Dispolex por problemas con el servicio del sitio que contiene el programa), por tanto, índices como la disponibilidad y la cohesión, las frecuencias relativa y acumulada, y el porcentaje de aparición no se pudieron obtener. Además, se ofrece una comparación de los errores ortográficos de los vocablos por centros de interés que constituye una útil información para la planificación de la docencia.

Sierra y Pérez a través del artículo "Disponibilidad léxica de estudiantes de español como lengua extranjera en Santiago de Cuba" (2016) socializan el análisis cuantitativo de la disponibilidad léxica de 50 estudiantes de ELE que asistieron a los cursos impartidos por la Universidad de Oriente y la Facultad de Medicina 2 de la Universidad de Ciencias Médicas, en Santiago de Cuba, entre octubre y noviembre de 2015. Las variables sociales empleadas y la estratificación de la muestra fueron: sexo (masculino: 28, femenino: 22), edad (jóvenes: 38, adultos mayores: 12), nivel de español (nivel elemental: 26, nivel intermedio: 24), conocimiento de otras lenguas (sí conocen: 25, no conocen: 25), lengua materna (francés/ creol.: 31, inglés: 11, italiano: 8). Se tuvieron en cuenta los 16 centros de interés tradicionales, lo que aportó 1786 vocablos y 7258 palabras. La media de vocablos por CI fue de 99.22, y por informante, de 35.72. Las áreas temáticas con mayor productividad de vocablos fueron la ciudad y profesiones y oficios; mientras que alimentos y el cuerpo bumano arrojaron mayor cantidad de palabras. Además, los que presentaron mayor índice de cohesión fueron medios de transporte y la ropa. Entre las conclusiones, las autoras mencionan la necesidad de apoyarse en estudios similares sobre hablantes nativos para determinar las necesidades léxicas de los no hispanohablantes y adecuar mejor sus cursos de español, así como la utilidad del promedio de respuestas por sujeto para dar cuenta de la efectividad del proceso de enseñanza-aprendizaje del léxico en estos estudiantes.

Los datos anteriores forman parte del estudio realizado por la primera autora para la tesis de maestría Selección del vocabulario para la enseñanza de ELE en Santiago de Cuba 
basada en la disponibilidad léxica (B1), la cual fue defendida en 2017. El objetivo general que se plantea en este trabajo es elaborar una metodología que permita la selección científica del vocabulario que debe ser enseñado en las clases de ELE (nivel B1) en Santiago de Cuba. La metodología, estructurada en cinco pasos, propone efectuar un estudio de disponibilidad léxica en hablantes nativos de la comunidad que será contexto de inmersión, así como en los estudiantes de ELE. A continuación, elegir las palabras de ambos listados que tienen un ID $\geq 0.05 \mathrm{y}$ han sido mencionadas por al menos el $10 \%$ de los informantes y comparar los resultados de ambos estudios de disponibilidad al aplicar este criterio de corte. Por último, contrastar los resultados con la Propuesta del Plan Curricular del Instituto Cervantes para concluir la selección. En la tesis se ofrece el diccionario temático comparativo: Léxico disponible de estudiantes nativos santiagueros y estudiantes de ELE, que a decir de la autora: "proporciona a profesores y estudiantes de ELE un material de consulta y apoyo para el perfeccionamiento de la enseñanza-aprendizaje del nivel lexical partiendo del léxico de uso real de la comunidad de habla en Santiago de Cuba y el léxico de estudiantes extranjeros" (Sierra 2017, p. 65). Finalmente, se recomienda el uso de los datos estadísticos que acompañan a las unidades léxicas como elemento del que debieran partir los responsables de la elaboración de aquellos materiales didácticos en los que interviene la selección léxica por niveles de estudio de la lengua.

\section{CONCLUSIONES}

El presente estudio ha permitido concluir que los seis años de investigaciones sobre disponibilidad léxica en Cuba, aquí referidos, muestran un interés creciente entre los lingüistas del país por desarrollar esta temática. En este corto periodo de tiempo se han logrado aportes en las tres regiones de la isla: occidental, central y oriental.

Puede afirmarse que la mayoría de los trabajos se adecuan a la metodología del Proyecto Panhispánico de Disponibilidad Léxica. No obstante, se recomienda una mayor uniformidad en los objetivos, informantes y métodos de selección de la muestra que permitan establecer comparaciones entre las regiones. 
Por último, se conoce sobre la existencia de cuatro tesis doctorales aún en desarrollo: en La Habana, que vincula la DL y la enseñanza del español como lengua extranjera; en Matanzas, que estudia el desarrollo léxico de estudiantes de Periodismo; en Las Tunas, ya mencionada; y en Santiago de Cuba, que caracteriza el vocabulario disponible de esa ciudad.

\section{BIBLIOGRAFÍA}

Alentado Echagarrua, A. (2017). Análisis y descripción de la disponibilidad léxica de los escolares de seis institutos preuniversitarios habaneros en tres centros de interés. Trabajo de Diploma, Universidad de La Habana, Cuba (Inédito)

Almeida Victorero, (2012). La enseñanza del español en el mundo hispánico. Disponibilidad léxica en escolares de La Habana. Trabajo de Diploma, Universidad de La Habana, Cuba (Inédito)

Ávila Muñoz, A. M. y Villena Ponsoda, J. A. (2010). Variación social del léxico disponible en la ciudad de Málaga. Diccionario y Análisis. Editorial Sarriá, S.L.

Bartol Hernández, J. A. (2006). La disponibilidad léxica, en Revista Española de Lingüirstica (RSEL) (36), pp. 379384. Disponible [en línea]: https://www.sel.edu.es/pdf/ene-dic-06/RSEL-36-Bartol.pdf.

Cabrera Albert, J. S., Pacheco Carpio, C. R., Rodríguez Vento, D. J. y González López, I. (2015). La disponibilidad léxica en estudiantes de preuniversitario de Pinar del Río, Cuba: Incidencia de la variable sexo. Ponencia presentada ante IX Conferencia Internacional Lingüistica 2015. Instituto de Literatura y Lingüística José Antonio Portuondo Valdor, La Habana, Cuba.

Cabrera Domecq, E. (2015). Índice de disponibilidad léxica en estudiantes de español como segunda lengua. Ponencia presentada ante IX Conferencia Internacional Lingüistica 2015. Instituto de Literatura y Lingüística José Antonio Portuondo Valdor, La Habana, Cuba.

Cabrera Domecq, E., Huo X. y Yun, S. (2015). La disponibilidad léxica en estudiantes sinohablantes de ELE en la carrera de Medicina. En Comunicación social: retos y perspectivas Vol. I. Santiago de Cuba: Ediciones Centro de Lingüística Aplicada, pp. 95-99.

Cabrera Domecq, E. y Chen, J. (2017). Análisis comparativo de la disponibilidad en español como lengua extranjera entre estudiantes chinos y sudafricanos. En Nuevos estudios sobre comunicación social, Vol. I, Santiago de Cuba: Ediciones Centro de Lingüística Aplicada, pp. 98-102.

Calzadilla Vega, G. y Domínguez Hernández, M. A. (2016). Competencia comunicativa, disponibilidad léxica y lectura. Sus relaciones en la formación inicial de profesores de Español- Literatura. En X Simposio Internacional Enseñanza-Aprendizaje de la Lengua y de la Literatura., Ciudad de México: Asociación Mexicana de Profesores de Lengua y Literatura. Disponible [línea]: https://www.ampll.org.mx/simposio.html. 
Cárdenas Rosales, S. (2016). La disponibilidad léxica en los estudiantes de onceno grado del IPVCE Ernesto Guevara en la provincia de Villa Clara. Trabajo de Diploma, Universidad Central Marta Abreu de Las Villas, Villa Clara, Cuba. Dispnible [en línea]: https://dspace.uclv.edu.cu/bitstream/handle/123456789/6482/Tesis $\% 20$ Sandra $\% 20 \mathrm{C} \% \mathrm{C} 3 \% \mathrm{~A}$ $\underline{1 \text { rdenas } \% 20 \text { Rosales } \% 20 \text { ok.pdf? sequence }=1 \text { \&is Allowed }=\mathrm{y}}$.

Cuba Vega, L. E. y Cabrera Domecq, E. (2013). El léxico de estudiantes sinohablantes de español. Estudios preliminares. En Actualizaciones en Comunicación Social. Vol. I, Santiago de Cuba: Ediciones Centro de Lingüística Aplicada, pp. 181-185.

Ecured (2018). https://www.ecured.cu/Batalla de ideas (23 de mayo de 2018).

Fernández Leyva, H. (2013). La selección y ocurrencias del vocabulario por centros de interés en el Manual Español para Todos 1. Tesis en opción al título de Máster en Enseñanza del Español como Lengua Extranjera. Universidad de Oriente, Santiago de Cuba, Cuba. (Inédita).

Fernández Leyva, H. y Pérez Marqués, C. M.a (2014). La disponibilidad léxica como recurso para el acercamiento a la identidad cultural en el aula de ELE. Ponencia presentada ante II Coloquio de Español como lengua extranjera. Universidad de La Habana, Cuba.

Fernández Leyva, H. (2015). Posible aplicación del índice de disponibilidad léxica a la selección del vocabulario de manuales de ELE, en Marcoele Revista de Didáctica de Español como Lengua Extranjera. (20), pp. 1-13. Disponible [en línea]: https://marcoele.com/descargas/20/fernandezdisponiblidad lexica.pdf.

Oramas Díaz, M. M. y García Caballero, Á. M.a (2017). Estudio de disponibilidad léxica de estudiantes de tercer año de bachillerato en las ciudades de Matanzas y Morelia (DLM2). Ponencia presentada ante la X Conferencia Internacional Lingü̈stica 2017, Estudios Lingüisticos. Instituto de Literatura y Lingüística José Antonio Portuondo Valdor, La Habana, Cuba.

Oramas Díaz, M. M. y García Caballero, Á. M. a (2018). Estudio de Disponibilidad Léxica en estudiantes de bachillerato de las ciudades de Matanzas y Morelia, en Atenas, (42/2), pp. 17-31. Disponible [en línea]: https://atenas.reduniv.edu.cu/index.php/atenas/article/view/368.

Pacheco Carpio, C. R., Cabrera Albert, J. S. y González López, I. (2017). Incidencia de la variable 'sexo' en la disponibilidad léxica de estudiantes de preuniversitario de Pinar del Río, Cuba, en Íkala (22/2), pp. 237-253. Disponible [en línea]: https://aprendeenlinea.udea.edu.co/revistas/index.php/ikala/article/viewFile/25809/20784948.

Perdomo Casanova, N., Hernández Pérez, M. y Sáez Cardenal L. (2015). La ortografía en el campo de interés 07: La cocina y sus utensilios, desde los estudios de disponibilidad léxica en estudiantes de duodécimo grado en la provincia de Matanzas. Ponencia presentada ante Humanística 2015. Matanzas, Cuba.

Perdomo Casanova, N., Alfonso Ramos, R. E. y Díaz Domínguez, B. H. (2017). Estudio de la disponibilidad léxica en el centro de interés 'Partes del cuerpo' en la provincia de Matanzas. Ponencia presentada ante la X Conferencia Internacional Lingüistica 2017, Estudios Lingüisticos. Instituto de Literatura y Lingüística José Antonio Portuondo Valdor, La Habana, Cuba. 
Pérez Marqués, C. M. a (2011). Disponibilidad léxica de los escolares de Guamá en tres centros de interés. En Comunicación Social en el siglo XXI, Vol. I, Santiago de Cuba: Ediciones Centro de Lingüística Aplicada, pp. 80-84.

Pérez Marqués, C. M.a (2012). Una visión regional a través de la disponibilidad léxica de los escolares del municipio Guamá. Ponencia presentada ante los Nuevos Talleres Internacionales de Estudios Regionales y Locales (Urbanos) III "La Región, sus Diversas Visiones”. Universidad Central de Las Villas, Santa Clara, Cuba.

Pérez Marqués, C. M. (2014). La disponibilidad léxica de los escolares del municipio Guamá como reflejo de su cultura local. En IX Conferencia Internacional sobre Lenguas Extranjeras, Comunicación y Cultura, WEFLA 2014. Universidad de Holguín, Cuba. Disponible [en línea]: https://sites.google.com/site/weflaholguin/system/app/pages/search?scope $=$ searchsite\&q $=\% \mathrm{C}$ 3\%A9rez+Marqu $\% \mathrm{C} 3 \% \mathrm{~A} 9 \mathrm{~s}$.

Pérez Marqués, C. M. ', Gómez Lujo, K., Álvarez Silva, R. M.a, Quintana Polanco, M. y Jackson Rodríguez D. (2015). Proyecto 'Vocabulario disponible de los estudiantes de noveno grado en Santiago de Cuba': un monitoreo de la efectividad del sistema educativo cubano desde la léxico-estadística. En Comunicación Social: retos y perspectivas, Vol. I, Santiago de Cuba: Ediciones Centro de Lingüística Aplicada, pp. 63-67.

Pérez Marqués, C. M. ${ }^{a}$, Muñoz Alvarado A., Quintana Polanco, M., Jackson Rodríguez, D. y Gómez Lujo, K. (2015). Disponibilidad de errores ortográficos en estudiantes de noveno grado de Santiago de Cuba. Ponencia presentada ante la IX Conferencia Internacional Lingüistica 2015. Instituto de Literatura y Lingǘstica José Antonio Portuondo Valdor, La Habana, Cuba.

Sierra Salas, L. G. (2013). Disponibilidad Léxica de Estudiantes Preuniversitarios Santiagueros. Trabajo de Diploma. Universidad de Oriente, Santiago de Cuba, Cuba (Inédito).

Sierra Salas, L. G. y Pérez Marqués, C. M. (2016). Disponibilidad léxica de estudiantes de español como lengua extranjera en Santiago de Cuba, en Santiago. (141), pp. 770-282. Disponible [en línea]: http://ojs.uo.edu.cu/index.php/stgo/article/viewFile/1601/1571.

Sierra Salas, L. G. (2017). Disponibilidad léxica de estudiantes de español como lengua extranjera en Santiago de Cuba. En Nuevos estudios sobre Comunicación Social, Vol. I, Santiago de Cuba: Ediciones Centro de Lingüística Aplicada, pp. 182-186.

Sierra Salas, L. G. (2017). Selección del vocabulario para la enseñanza de ELE en Santiago de Cuba basada en la disponibilidad léxica (B1). Tesis en opción al título académico de Máster en Enseñanza del Español como Lengua Extranjera. Universidad de Oriente, Santiago de Cuba, Cuba (Inédita).

Suardíaz del Río, L. (2015). "Léxico disponible de los escolares habaneros y matanceros en dos centros de interés: descripción, análisis y comparación”. Ponencia presentada ante la IX Conferencia Internacional Lingüistica 2015. Instituto de Literatura y Lingüística José Antonio Portuondo Valdor, La Habana, Cuba. 